\title{
Hipocalcemia severa o sintomática secundaria a hipoparatiroidismo posoperatorio en cirugía de tiroides: experiencia en un hospital universitario de Medellín, Colombia
}

\author{
Severe or symptomatic hypocalcemia due to postoperative \\ hypoparathyroidism in thyroid surgery at a reference hospital in Medellín, \\ Colombia
}

\author{
Buriticá González J, ${ }^{1}$ Muñoz Ríos JH, ${ }^{1}$ Buitrago Giraldo $N,{ }^{1}$ Arias Velásquez CA, ${ }^{1}$ \\ Betancur Echavarría AC, ${ }^{1}$ Pizza MJ, ${ }^{2}$ Aristizábal Henao $N^{3}$
}

${ }^{1}$ Estudiantes pregrado de Medicina. Universidad Pontificia Bolivariana. Medellín, Colombia.

${ }^{2}$ Residente Medicina Interna. Universidad Pontificia Bolivariana. Medellín, Colombia.

${ }^{3}$ Medicina Interna. Endocrinología, Diabetes y Metabolismo. Clínica Las Américas. Medellín, Colombia.

Autor de correspondencia: Natalia Aristizábal Henao

Correo electrónico: naty_aristizabal@yahoo.com

Fecha de recepción: 9/12/2019

Fecha de aceptación: 8/02/2020

\section{Resumen}

Objetivo: caracterizar la población de pacientes que presentan hipocalcemia severa o sintomática después de una tiroidectomía total y que requieren calcio parenteral.

Diseño: estudio observacional retrospectivo realizado en un centro especializado de Medellín, Colombia.

Marco de referencia: la hipocalcemia posoperatoria (POP) es una complicación bien reconocida de la tiroidectomía, que se caracteriza por la presencia de hipocalcemia, con niveles de hormona paratiroidea (Parathyroid hormone, PTH) bajos o inadecuadamente normales. La hipocalcemia sintomática o severa (calcio corregido $<7,5 \mathrm{mg} / \mathrm{dL}$ ) es una verdadera emergencia médica, que requiere un rápido diagnóstico y tratamiento con calcio parenteral.

Pacientes: pacientes en POP de tiroidectomía que presentan hipocalcemia severa o sintomática.
Intervenciones: reposición con calcio parenteral.

Resultados: la hipocalcemia severa o sintomática se presentó en el 8 \% de los pacientes llevados a una tiroidectomía total, con predominio en el sexo femenino. La patología tiroidea maligna se constituyó en la indicación más frecuente de la cirugía. En estos pacientes, la media de la PTH fue de 11,3 pg/ $\mathrm{mL}$, mientras que los valores de calcio más bajos se presentaron a las 48 horas POP. Solo se visualizaron las paratiroides en cirugía en una tercera parte de los casos y un paciente tuvo una reintervención en las primeras 24 horas; hasta el $18 \%$ tuvieron hipomagnesemia concomitante.

Conclusión: la hipocalcemia por hipoparatiroidismo POP es una complicación frecuente después de la cirugía de tiroides, y en un grupo de pacientes será severa o sintomática, razón por la cual requiere uso de calcio parenteral. En consecuencia, esta complicación tendrá que identificarse y tratarse como una emergencia médica para disminuir la morbilidad y la potencial mortalidad asociadas.

Palabras clave: hipocalcemia, hipoparatiroidismo, tiroidectomía.

\section{Abstract}

Objective: the aim of this work was to describe characteristics of patients with severe or symptomatic hypocalcemia after total thyroidectomy who required treatment with parenteral calcium.

Methods: the authors conducted an observational retrospective analysis at a reference hospital in Medellín, Colombia. 
Background: postoperative hypocalcemia is a frequent complication after thyroidectomy; it is characterized by the presence of hypocalcemia with low or normal values of parathyroid hormone. Symptomatic or severe hypocalcemia (serum calcium $<7.5 \mathrm{mg} / \mathrm{dL}$ ) is a medical emergency and require early diagnostic and treatment with parenteral calcium.

Patients: Patients with severe or symptomatic hypocalcemia after total thyroidectomy.

Intervention: treatment with parenteral calcium.

Results: symptomatic or severe hypocalcemia was present in $8 \%$ of patients who went to total thyroidectomy, most of patients were female, and thyroid cancer was main indication for surgery; the average of parathyroid hormone (PTH) value was $11.3 \mathrm{pg} / \mathrm{mL}$ and the lowest calcium value was present at 48 hours in the postoperative period. Parathyroid glands only were visualized in one third of these patients and just one patient had second surgery in the first 24 hours; $18 \%$ of patients had hypomagnesemia associated with hypocalcemia.

Conclusion: postoperative hypocalcemia due to hypoparathyroidism is a frequent complication after thyroid surgery; some of these patients will develop symptomatic or severe hypocalcemia and require parenteral calcium. It is important identify and treat this condition as an emergency with the propose of diminishing associated morbidity and mortality.

Keywords: hypocalcemia, hypoparathyroidism, thyroidectomy.

\section{Introducción}

La hipocalcemia posoperatoria (POP) es una complicación bien reconocida de la tiroidectomía total, que se caracteriza por la presencia de hipocalcemia, con niveles de hormona paratiroidea (PTH) bajos o inadecuadamente normales $(1,2)$. Su prevalencia es variable y oscila entre el 10 y el $83 \%(1,3-9)$. Esto se explica por la técnica quirúrgica y las diferentes definiciones usadas. Así, la hipocalcemia sintomática ocurre en el 10 al $36 \%$ de los casos (4) y constituye una emergencia médica, pues es una complicación clínica potencialmente grave (1), y la hipomagnesemia es un factor de riesgo independiente para este trastorno hidroelectrolítico (4). Entre tanto, la incidencia de la hipocalcemia transitoria (duración $<6-12$ meses) $(1,10)$ es del $27 \%$ (19-38\%) y la hipocalcemia permanente alcanza el $1 \%(0-3 \%)(10)$, lo cual acarrea una morbilidad significativa y costos altos asociados a largo plazo (3).

La lesión de las glándulas paratiroides (trauma mecánico o térmico) está relacionada con la técnica quirúrgica y la extensión de la tiroidectomía, dado que estas deben ser identificadas in situ, manipuladas cuidadosamente y preservadas con su vascularización $(3,5)$. Esta lesión es entonces más frecuente en tiroidectomías totales y en reintervenciones quirúrgicas (11). El conocimiento de la anatomía del cuello y la experiencia del cirujano son fundamentales para disminuir el hipopa- ratiroidismo POP (3). Sin embargo, debe reconocerse que aun cuando se identifican las glándulas y son preservadas durante la cirugía, la función de las paratiroides en el POP no está garantizada (12), dado que la manipulación en la vecindad del tejido paratiroideo puede comprometer de forma transitoria la vascularización y causar aturdimiento (3).

Se han descrito múltiples protocolos para prevenir o identificar la hipocalcemia POP, los cuales incluyen la suplementación de calcio oral, las mediciones seriadas de calcio sérico y la monitorización de la PTH POP $(7,13-18)$. Esto permite extremar la vigilancia en los casos de alto riesgo de hipocalcemia sintomática POP (enfermedad de Graves, grandes bocios, reintervenciones o confirmación de la extirpación de alguna de las glándulas paratiroides) (1). La identificación rápida de la hipocalcemia puede evitar la aparición de síntomas, así como acortar la estancia hospitalaria y reducir los costos asociados (3). Además, la medición de la PTH el día después de la cirugía permite predecir la función paratiroidea POP y contribuye a reconocer a los pacientes que requieren suplementación con calcio y vitamina $\mathrm{D}(1,7,19)$.

En este contexto, la suplementación profiláctica con calcio oral se ha propuesto como un método para prevenir la hipocalcemia temprana POP (7). De hecho, la suplementación rutinaria con calcio constituye una medida efectiva a fin de continuar el manejo ambulatorio de los pacientes de forma segura y evitar la aparición de los síntomas de hipocalcemia (7). Según la gravedad del cuadro, el manejo consiste en la administración de calcio vía oral o intravenoso asociado al calcitriol oral (1, 20). El tratamiento por vía parenteral está indicado en pacientes con síntomas (anestesia perioral, parestesias en manos y pies) o niveles de calcio sérico corregidos $<7,5 \mathrm{mg} / \mathrm{dL}$ (3) (hipocalcemia severa) buscando metas de calcio sérico corregidos en el límite inferior de lo normal (3). El calcio intravenoso en bolos incrementa el calcio en la sangre solo durante 2 a 3 horas, de forma que se requieren infusiones continuas ajustadas a los valores de calcio medidos cada 6 a 8 horas (3) bajo monitorización electrocardiográfica continua (6), asociadas a la reposición con sales de calcio por vía oral. El carbonato de calcio es el que se usa con mayor frecuencia a dosis de 1 a $3 \mathrm{~g}$ de calcio elemental por día, dividido en 3-4 dosis administradas con las comidas, $(3,6)$ y asociadas al calcitriol, que es la forma activa de la vitamina D (3). No se debe olvidar la importancia de descartar la hipomagnesemia asociada y tratarla según sea el caso $(3,4)$.

El propósito de este estudio es caracterizar la población de pacientes que presentan hipocalcemia severa o sintomática después de una tiroidectomía total y requerimiento de calcio parenteral. Es necesario considerar que esta es una verdadera emergencia endocrina, por lo que debe reconocerse rápidamente y tratarse de forma adecuada, a fin de disminuir la morbilidad y la mortalidad asociadas. 


\section{Métodos}

Se trata de un estudio observacional retrospectivo, el cual se realizó en un centro especializado de Medellín (Colombia). Se incluyeron todos los pacientes adultos (mayores de 18 años) llevados a tiroidectomía total por parte del Servicio de Cirugía de Cabeza y Cuello y quienes presentaron hipocalcemia POP severa (calcio sérico corregido $<7,5 \mathrm{mg} / \mathrm{dL}$ ) o sintomática (ocurrencia de parestesias en extremidades, anestesia perioral, signos de Chvostek y Trousseau, intervalo QTc prolongado, coma, convulsión, falla cardíaca) entre diciembre de 2015 y diciembre de 2017.

Se recogieron variables sociodemográficas, antropométricas, clínicas y de laboratorio, mientras que la historia clínica fue considerada la principal fuente de información para el estudio. Los datos que eventualmente no se encontraron en este documento fueron extraídos de los registros de laboratorio.

De forma independiente, un investigador revisó los criterios de elegibilidad de los pacientes y la información registrada acerca de las variables de interés, en un instrumento de recolección diseñado con este fin. Otro investigador verificó las inconsistencias entre la información de la historia clínica. Luego de ello, los datos fueron ingresados en una base de datos de Microsoft Excel y analizados mediante IBM SPSS.

Se realizó un análisis descriptivo de las variables de estudio, a partir de medidas como frecuencias absolutas y relativas para variables cualitativas (sexo, indicación quirúrgica, reintervención en 24 h, visualización de paratiroides, hipomagnesemia, forma farmacéutica de presentación de calcio, hipocal- cemia sintomática y síntomas asociados). Para las variables cuantitativas (edad, tiempo quirúrgico en horas, duración de la infusión de calcio, dosis de calcio elemental, dosis de calcitriol, creatinina, albúmina, magnesio, fósforo, calcio a las 6, 12, 24, 36 y 48 horas, PTH POP) se utilizaron promedios y desviación estándar (DS).

El protocolo de investigación fue evaluado y aprobado por el comité de ética del centro especializado.

\section{Resultados}

En el período comprendido entre diciembre de 2015 y diciembre de 2017 en un hospital universitario de Medellín, Antioquia, 408 pacientes fueron llevados a cirugía de cabeza y cuello, de los cuales a 275 se les realizó una tiroidectomía total (Tabla 1); en este grupo, 22 individuos (8\%) presentaron hipocalcemia aguda severa o sintomática.

La duración media de las cirugías de tiroides fue de 3,5 h (DS: 2,55$)$ y un paciente requirió de una reintervención quirúrgica en las primeras 24 horas POP por sangrado agudo. Solo en el 36,4 \% de estos pacientes se visualizaron las paratiroides. Entre tanto, la media de PTH POP fue de $11,3 \mathrm{pg} / \mathrm{mL}$ (DS: 17,66), mientras que los valores más bajos de calcio fueron reportados a las 36 horas POP (6,8 mg/dL DS: 1,2$)$ y la hipomagnesemia $(<1,6 \mathrm{mg} / \mathrm{dL})$ concomitante ocurrió en el $18 \%$ de los pacientes con hipocalcemia POP severa o sintomática.

Al evaluar la indicación de calcio parenteral, encontramos 10 pacientes sintomáticos (45,5\%) y 12 con hipocalcemia severa no sintomática (calcio corregido $<7,5 \mathrm{mg} / \mathrm{dL}$ ). En los

Tabla 1. Pacientes que fueron llevados a cirugía de cabeza y cuello. Se identifican aquellos con hipocalcemia y requerimiento de calcio parenteral después de la tiroidectomía total

\begin{tabular}{l|c}
\multicolumn{1}{c}{$\begin{array}{c}\text { Cirugías entre diciembre de } \mathbf{2 0 1 5} \text { y diciembre de 2017 en } \\
\text { Hospital Universitario de Medellín Antioquia. }\end{array}$} \\
$\begin{array}{l}\text { Características de los pacientes llevados a tiroidectomía total con hipocalcemia aguda } \\
\text { sintomática o severa con requerimiento de calcio intravenoso }\end{array}$ & Número de pacientes (\%) \\
\hline $\begin{array}{l}\text { Total de pacientes con hipocalcemia aguda sintomática o severa (calcio <7,5 mg/dL) en el pos- } \\
\text { operatorio con requerimiento de calcio intravenoso }\end{array}$ & $22 / 257(8,5 \%)$ \\
\hline Edad promedio de los pacientes & 58 años (DS: 16) \\
\hline Mujeres & $19 / 22(86,36 \%)$ \\
\hline $\begin{array}{l}\text { Indicaciones de tiroidectomía total: enfermedad tiroidea maligna } \\
\text { Bocio multinodular con síntomas compresivos }\end{array}$ & $14 / 22(63,63 \%)$ \\
\hline Tirotoxicosis de difícil control & $6 / 22(27,27 \%)$ \\
\hline $\begin{array}{l}\text { Pacientes con requerimiento de reintervención quirúrgica en las primeras 24 horas posoperato- } \\
\text { rias por sangrado agudo }\end{array}$ & $2 / 22(9,09 \%)$ \\
\hline
\end{tabular}


casos de hipocalcemia severa asintomática se indicó el inicio de una infusión de calcio sin uso de bolo, la cual se preparó de tal forma que cada mililitro (mL) de la solución aportaba 1 miligramo (mg) de calcio elemental, lo que corresponde a una dosis de $1 \mathrm{mg} / \mathrm{kg}$ de calcio elemental que se tituló de acuerdo con los controles de calcio sérico medidos cada 6 horas. En los pacientes sintomáticos se indicó el uso de un bolo de gluconato de calcio ( 1 ampolla de gluconato de calcio al $10 \%$, diluida en $50 \mathrm{~mL}$ de dextrosa en agua destilada [DAD] al $5 \%$ en 20 minutos) con posterior inicio de la infusión mencionada.

El suplemento de calcio oral se inició de manera simultánea al aporte parenteral. La principal forma de presentación usada fue el carbonato de calcio en 20 pacientes y solo en 2 de ellos se administró citrato de calcio por intolerancia gastrointestinal. Se requirieron, en promedio, 5,133 g de calcio elemental cada día y 0,7 $\mu \mathrm{g}$ de calcitriol (dosis mínima de 0,25 $\mu \mathrm{g}$ y máxima $1 \mu \mathrm{g} / \mathrm{d}$ ).

\section{Discusión}

La hipocalcemia es una de las principales complicaciones POP de las cirugías de cabeza y cuello. Las tiroidectomías totales son cirugías frecuentemente realizadas en todo el mundo y la mayoría de los pacientes llevados a este tipo de intervención son mujeres (83\%) como se observó en nuestro estudio, en el cual se encontró una predominancia de pacientes del sexo femenino (86,4 \%) (7). Aunque en nuestro caso la indicación más común para la intervención quirúrgica fue la patología tiroidea maligna, en otras series ha sido el bocio multinodular $(64,1 \%)(1-3)$.

De otro lado, la hipomagnesemia ha sido reportada en el $23,4 \%$ de los pacientes, luego de haberse realizado la tiroidectomía, y constituye un factor de riesgo independiente para la ocurrencia de hipocalcemia (4). En nuestros pacientes con requerimiento de reposición de calcio parenteral se encontró esta alteración hidroelectrolítica en el 18 \%, lo que coincidió con lo reportado en la literatura.

La falla en la identificación de las glándulas paratiroides durante la tiroidectomía puede incrementar el riesgo de lesión y de resección inadvertida (3). Asimismo, la prevalencia de la hipocalcemia es inversamente proporcional a la permanencia de las glándulas paratiroides in situ (21). En efecto, algunos consensos recomiendan la búsqueda e identificación de todas las glándulas paratiroides durante la cirugía (1). Sin embargo, datos recientes reportados en una cohorte con un menor número de pacientes evidencian que la identificación rutinaria y sistemática de las cuatro glándulas paratiroides (lo cual puede ser un reto peligroso, dado el riesgo de traumatizar más tejido o de generar su vascularización) parece no ser necesaria en las tiroidectomías realizadas mediante disección capsular.

Esto se debe a que no existe diferencia en la incidencia de la hipocalcemia clínica en los casos en los cuales se identifican
0-2 glándulas, en comparación con 3-4 glándulas, y tampoco hay diferencia en las paratiroidectomías inadvertidas (5), dado que es probable que la importancia de la identificación de las glándulas paratiroides esté influenciada por la técnica usada durante la cirugía (5). Se incrementa la hipocalcemia transitoria no permanente cuando 3 o menos glándulas paratiroides son preservadas $(3,22)$. En este reporte, solo se visualizaron las paratiroides durante la cirugía en una tercera parte de los pacientes que presentaron hipocalcemia grave o sintomática. Entre tanto, los predictores independientes de hipocalcemia permanente que han sido identificados son las reintervenciones por sangrado y la enfermedad de Graves como indicación de tiroidectomía total (3). Por otro lado, según un metaanálisis la enfermedad de Graves (odds ratio [OR]: 1,75) y el sexo femenino (OR: 2,28) se han encontrado como factores asociados a la hipocalcemia transitoria (11).

En los casos de hipertiroidismo, el aumento del tamaño de la glándula tiroidea y su vascularización parecen explicar la dificultad en la localización y la preservación de las paratiroides (3). Solo 2 de los pacientes de la serie aquí reportada fueron llevados a cirugía por tirotoxicosis no controlada.

La medición de la PTH intacta en el POP parece ser una medida eficiente y costoefectiva en el diagnóstico del hipoparatiroidismo POP y la predicción de hipocalcemia $(14-17,19)$. Así pues, los valores bajos de la PTH intacta $(<6-35 \mathrm{pg} / \mathrm{mL}$, el día 1 POP) tienen una sensibilidad del 69 al $100 \%$ para hipocalcemia transitoria en el POP $(3,17)$. También los niveles bajos de vitamina D3 preoperatorios aumentan el riesgo en el POP (11), así como lo hace la hipomagnesemia (3), tal y como ya se había mencionado.

Para el diagnóstico de la hipocalcemia POP no se han encontrado diferencias estadísticamente significativas en el momento de realizar la PTH intacta (7). Esto sugiere que los niveles séricos de PTH intacta en el POP, a las 12 y 24 horas, pueden predecir el desarrollo de una hipocalcemia POP (7). Algunos autores han sugerido que el cambio o gradiente de la PTH intacta puede ser más útil que su valor POP aislado en la predicción del riesgo de hipocalcemia (23). Esta habitualmente ocurre entre las 24 y 48 horas POP (7), aunque el nadir en los niveles de calcio y la aparición de síntomas pueden diferirse hasta 48-72 horas desde la cirugía. Por tanto, se han propuesto diferentes métodos para predecir la aparición clínica de la hipocalcemia tras una tiroidectomía que incluyen mediciones seriadas de calcio y paratohormona en el posoperatorio (1).

La suplementación profiláctica con calcio oral puede prevenir la hipocalcemia POP temprana, y es una medida costoefectiva y segura (7). Sin embargo, algunos consensos no recomiendan la administración profiláctica de calcio y vitamina D en el preoperatorio, excepto en los casos con evidencia de déficit en este período (1). Llaman la atención los altos requerimientos de calcio oral que se encontraron en el estudio realizado, los cuales superaron las recomendaciones de la li- 
teratura (alrededor de $3 \mathrm{~g}$ de calcio elemental). No obstante, dado que solo se analizó la ocurrencia de hipocalcemia en el POP inmediato, no es posible evaluar cómo se titularon las dosis a largo plazo, qué eventos adversos se pudieron asociar a esta suplencia de calcio oral, ni el porcentaje de pacientes con hipocalcemia persistente en el tiempo.

En vista del carácter retrospectivo del estudio, el subregistro de datos en la historia clínica, principal fuente de información, se presenta como una limitación. Esto invita a la realización y el diseño de estudios prospectivos con un adecuado diligenciamiento de las bases de datos.

\section{Conclusión}

La hipocalcemia secundaria a hipoparatiroidismo POP es una complicación frecuente después de la cirugía de tiroides.
En un grupo de pacientes, esta condición será severa o sintomática y requerirá calcio parenteral. Por tanto, tendrá que identificarse y tratarse como una emergencia médica, a fin de disminuir la morbilidad y la potencial mortalidad asociada.

\section{Conflicto de intereses}

Los autores declaran no tener conflictos de interés.

\section{Financiación}

El artículo no cuenta con fuentes de financiación institucionales. Hace parte del compromiso académico la Asociación Colombiana de Endocrinología para obtener patrocinio y presentar los resultados preliminares de este trabajo en forma de póster, durante el Congreso Europeo de Tiroides ( $42^{\text {nd }}$ Annual Meeting of the European Thyroid Association).

\section{Referencias}

1. Castro A, Oleaga A, Parente P, Paja M, Gil E, Álvarez C. Resumen ejecutivo del documento de consenso SEORL CCC-SEEN sobre hipoparatiroidismo postiroidectomía. Endocrinol Diabetes Nutr. 2019;66(7):459-463.

2. Shoback DM, Bilezikian JP, Costa AG, Dempster D, Dralle H, Khan AA, et al. Presentation of Hypoparathyroidism: Etiologies and Clinical Features. J Clin Endocrinol Metab. 2016;101(6):2300-12.

3. Dedivitis RA, Aires FT, Cernea CR. Hypoparathyroidism after thyroidectomy: prevention, assessment and management. Curr Opin Otolaryngol Head Neck Surg. 2017;25(2):142-146.

4. Luo H, Yang H, Zhao W, Wei T, Su A, Wang B, et al. Hypomagnesemia predicts postoperative biochemical hypocalcemia after thyroidectomy. BMC Surg. 2017;17(1):62.

5. Sheahan P, Mehanna R, Basheeth N, Murphy MS. Is systematic identification of all four parathyroid glands necessary Turing total thyroidectomy?: a prospective study. Laryngoscope. 2013;123(9):2324-8.

6. González J, Piedrahita D. Hypocalcaemia after total thyroidectomy: incidence, control and treatment. Acta Otorrinolaringol Esp. 2013;64(2):102107.

7. Arer IM, Kus M, Akkapulu N, Aytac HO, Yabanoglu H, Caliskan K, et al. Prophylactic oral calcium supplementation therapy to prevent early post thyroidectomy hypocalcemia and evaluation of postoperative parathyroid hormone levels to detect hypocalcemia: A prospective randomized study. Int J Surg. 2017;38:9-14.

8. Caglià $\mathrm{P}$, Puglisi S, Buffone $\mathrm{A}$, Bianco SL, Okatyeva V, Veroux M, et al. Post-thyroidectomy hypoparathyroidism, what should we keep in mind? Ann Ital Chir. 2017;6:371-381.

9. Martin S, Parfeni O, Mustata T, Andrei M, Sirbu A, Barbu C, et al. Postoperative Hypoparathyroidism in Patients After Total Thyroidectomy - Experience of a Tertiary Center in Romania. Chirurgia (Bucur). 2019;114(5):602610.

10. Almquist M, Hallgrimsson P, Nordenström E, Bergenfelz A. Prediction of permanent hypoparathyroidism after total thyroidectomy. World J Surg. 2014;38(10):2613-2620.

11. Edafe O, Antakia R, Laskar N, Uttley L, Balasubramanian SP. Systematic review and meta-analysis of predictors of post-thyroidectomy hypocalcaemia. Br J Surg. 2014;101(4):307-320.

12. Kakava K, Tournis S, Papadakis G, Karelas I, Stampouloglou P, Kassi E, et al. Postsurgical Hypoparathyroidism: A Systematic Review. In Vivo. 2016;30(3):171-9.
13. Bove A, Di Renzo RM, Palone G, D’Addetta V, Percario R, Panaccio P, et al. Early biomarkers of hypocalcemia following total thyroidectomy. Int J Surg. 2014;12 Suppl 1:S202-4.

14. Pisanu A, Saba A, Coghe F, Uccheddu A. Early prediction of hypocalcemia following total thyroidectomy using combined intact parathyroid hormone and serum calcium measurement. Langenbecks Arch Surg. 2013;398(3):423-430.

15. Graciano AJ, Chine CT, Fischer CA. Applicability of imediate, late or seria intact parathyroid hormone measurement following total thyroidectomy. Braz J. Otorhinolaryngol. 2012;78(5):78-82.

16. Lang $\mathrm{BH}, \mathrm{Yih} \mathrm{PC}, \mathrm{Ng} \mathrm{KK}$. A prospective evaluation of quick intraoperative parathyroid hormone assay at the time of skin closure in predicting clinically relevant hypocalcemia after thyroidectomy. World J Surg. 2012;36(6):1300-1306.

17. Castro A, Del Rio L, Gavilan J. Stratifying the Risk of Developing Clinical Hypocalcemia after Thyroidectomy with Parathyroid Hormone. Otolaryngol Head Neck Surg. 2018;158(1):76-82.

18. Orloff LA, Wiseman SM, Bernet VI, Fahey TJ, Shaha AR, Shindo ML, et al. American Thyroid Association Statement on Postoperative Hypoparathyroidism: Diagnosis, Prevention, and Management in Adults. Thyroid. 2018;28(7):830-841.

19. Selberherr A, Scheuba C, Riss P, Niederle B. Postoperative hypoparathyroidism after thyroidectomy: efficient and cost-effective diagnosis and treatment. Surgery. 2015;157(2):349-353.

20. Brandi ML, Bilezikian JP, Shoback D, Bouillon R, Clarke BL, Thakker RV, et al. Management of Hypoparathyroidism: Summary Statement and Guidelines. J Clin Endocrinol Metab. 2016;101(6):2273-83.

21. Lorente-Poch L, Sancho JJ, Ruiz S, Sitges-Serra A. Importance of in situ preservation of parathyroid glands during total thyroidectomy. Br J Surg. 2015;102(4):359-367.

22. Song CM, Jung JH, Ji YB, Min HJ, Ahn YH, Tae K. Relationship between hypoparathyroidism and the number of parathyroid glands preserved during thyroidectomy. World J Surg Oncol. 2014;12:200.

23. Al Khadem MG, Rettig EM, Dhillon VK, Russell JO, Tufano RP. Postoperative IPTH compared with IPTH gradient as predictors of post-thyroidectomy hypocalcemia. Laryngoscope. 2018;128(3):769-774. 


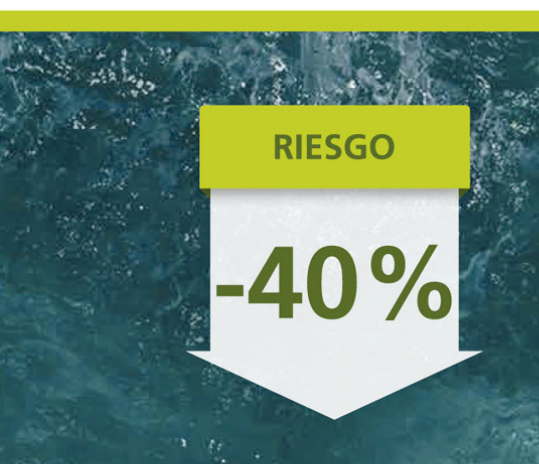

\section{REDUZCA UN $40 \%$ EL RIESGO DE HIPOGLECEMIA SEVERA DE SUS PACIENTES CON TRESIBA ${ }^{\circledR}$}

VS. GLARGINA U100

DÉ A SUS PACIENTES LA PREDICTIBILIDAD DIARIA QUE NECESITAN

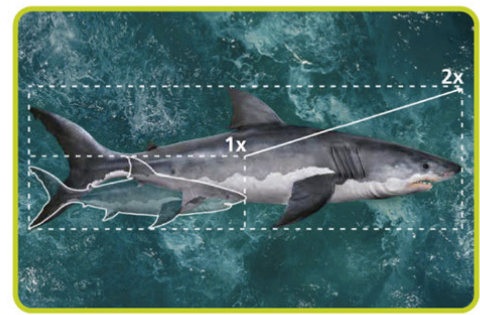

+ IMPACTO EN RIESGO CV

EL RIESGO DE MUERTE CV SE

DUPLICA DESPUÉS DE UN EPISODIO HIPOGLUCÉMICO SEVERO ${ }^{4 \dagger}$

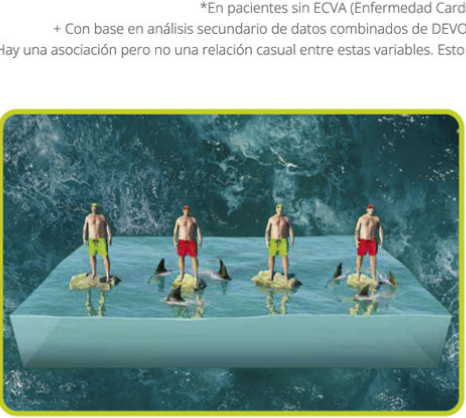

4

PREVALENCIA

APROXIMADAMENTE UNO DE CADA DOS PACIENTES TIENE AL MENOS UN EPISODIO HIPOGLUCÉMICO EN UN SOLO MES ${ }^{5 \dagger}$

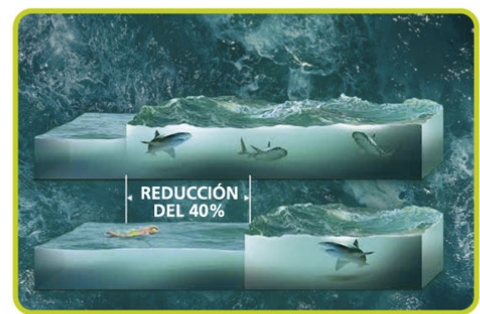

+ HipOGLUCEMIA

REDUCCIÓN DEL 40\% EN HIPOGLUCEMIA SEVERA CON TRESIBA ${ }^{\circledR}$ CON GLARGINA U100 EN COMPARACIÓN CON GLARGINA U1001§ 